\title{
A weighted simplified point score for prediction of difficult facemask ventilation - a cohort study
}

\author{
Lars H Lundstrøm'; Charlotte V Rosenstock1; -Jørn Wetterslev2; Anders K Nørskov1,2
}

1) Department of Anaesthesiology, Copenhagen University Hospital, Nordsjællands Hospital, Hillerød

2) Copenhagen Trial Unit, Centre for Clinical Intervention Research, Copenhagen University Hospital, Rigshospitalet

\section{Background and Goal of Study}

Difficult face mask ventilation (DMV) is associated with increased risk of complications. Our aim was to 1) assess risk factors associated with DMV and 2) evaluate the diagnostic accuracy of an accumulated weighted risk score for predicting DMV.

\section{Materials and Methods:}

A cohort of 46804 adults undergoing facemask ventilation was retrieved from the intervention group of the cluster randomized Difficult Airway Management Trial (DIFFICAIR) (1). Patients were airway assessed using 13 defined predictors for difficult airway management. The associations between DMV (2) and the 13 predictors were assessed by logistic regression and converted to a weighted simplified point score. The model was built from a population with complete data of the predictors in two consecutive steps. Initially, all the predefined variables were included in a multivariate regression analysis and the final model was derived by backward elimination at $P<0.05$. Secondly, the regression coefficients (beta-values) of the risk factors from the final logistic regression model were converted to a clinically applicable weighed simplified point score (3). The predictive ability of both the original regression equation using the betavalues and the simplified point score was evaluated by discrimination (area under the receiver operation curve (AUC). The optimal cutoff value of the score was calculated by the Youden index (sensitivity + specificity - 1).

Table 2. Diagnostic accuracy of a simplified point scores for difficult mask ventilation

\begin{tabular}{|c|c|c|c|c|c|c|c|c|}
\hline $\begin{array}{c}\text { Dichotomous cut-off } \\
\text { level }\end{array}$ & Sens. & Spec. & Pos. LR. & $\begin{array}{l}\text { Youden } \\
\text { index. }\end{array}$ & $\begin{array}{l}\text { Prev. of } \\
\text { DMV }\end{array}$ & $\begin{array}{l}\text { Odds } \\
\text { ratio }\end{array}$ & $95 \%$ CI & p-value \\
\hline Sum score $=1$ points & 0.99 & 0.11 & 1.1 & 0.100 & $1.2 \%$ & 10.8 & $4.0-28.9$ & $<0.0001$ \\
\hline Sum score $=2$ points & 0.99 & 0.18 & 1.2 & 0.169 & $1.3 \%$ & 15.4 & $6.4-37.2$ & $<0.0001$ \\
\hline Sum score $=3$ points & 0.98 & 0.25 & 1.3 & 0.235 & $1.4 \%$ & 19.1 & $8.5-42.9$ & $<0.0001$ \\
\hline Sum score $=4$ points & 0.97 & 0.37 & 1.5 & 0.332 & $1.7 \%$ & 16.2 & $9.1-28.8$ & $<0.0001$ \\
\hline Sum score $=5$ points & 0.94 & 0.49 & 1.8 & 0.429 & $2.0 \%$ & 14.9 & $9.6-23.2$ & $<0.0001$ \\
\hline Sum score $=6$ points & 0.85 & 0.61 & 2.2 & $0.461^{\mp}$ & $2.4 \%$ & 8.9 & $6.6-12.0$ & $<0.0001$ \\
\hline Sum score $=7$ points & 0.72 & 0.75 & 2.8 & $0.461^{*}$ & $3.0 \%$ & 7.4 & $5.8-9.3$ & $<0.0001$ \\
\hline Sum score $=8$ points & 0.56 & 0.86 & 3.9 & 0.420 & $4.2 \%$ & 7.8 & $6.3-9.6$ & $<0.0001$ \\
\hline Sum score $=9$ points & 0.44 & 0.93 & 5.8 & 0.361 & $6.1 \%$ & 9.6 & $7.7-11.9$ & $<0.0001$ \\
\hline Sum score $=10$ points & 0.29 & 0.97 & 8.2 & 0.250 & $8.4 \%$ & 11.1 & $8.7-14.1$ & $<0.0001$ \\
\hline
\end{tabular}

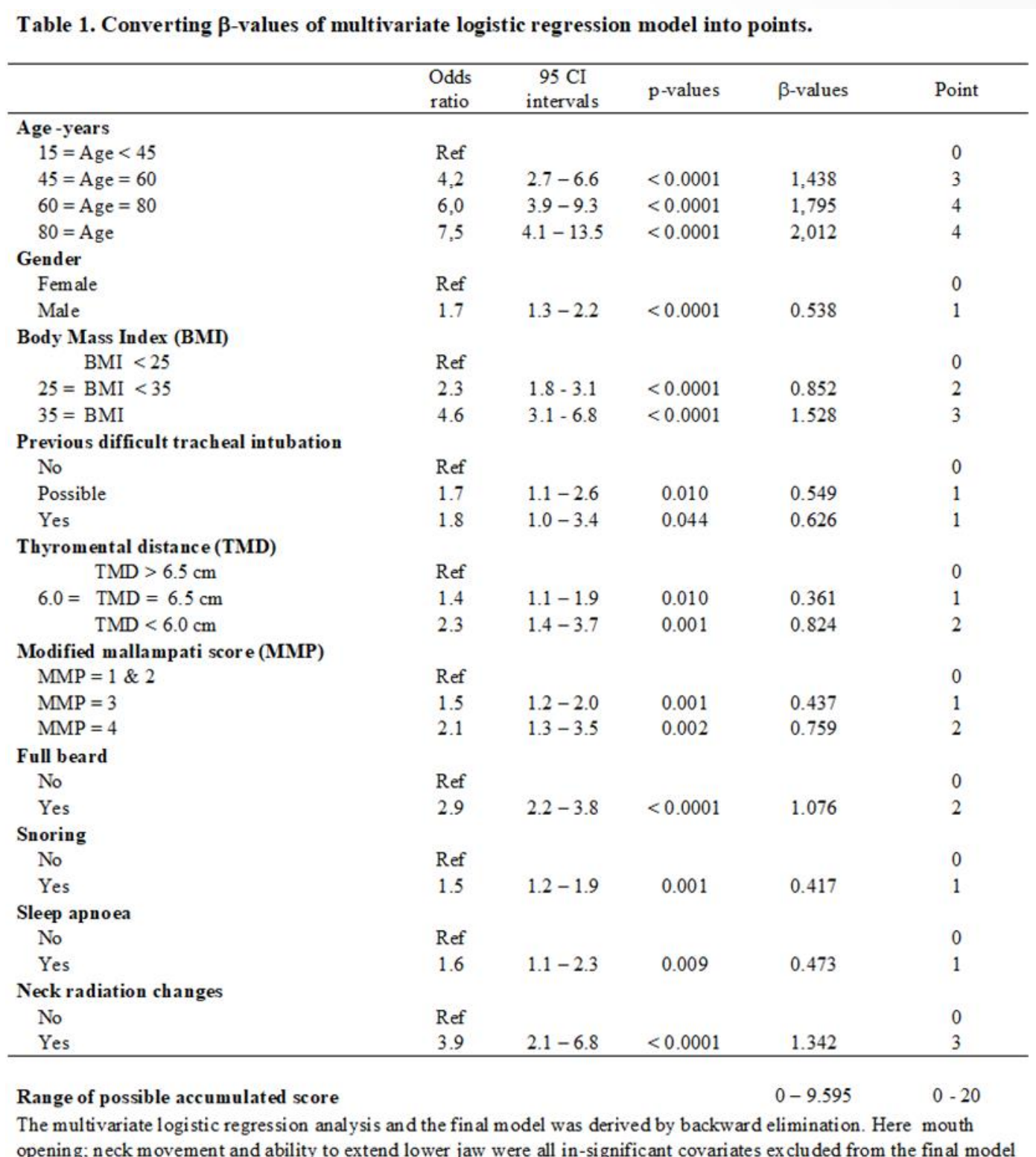

Results and Discussion:

The prevalence of DMV was 1.1\% $(1.0-1.2 \%, 95 \% \mathrm{Cl})$. High age, increased body mass index, and previous radiation of the neck were especially associated with DMV (Table 1). The conversion of beta-values into points for the weighted simplified point score is presented in table 1. The weighted simplified point score range from 0 to 20 points. The diagnostic accuracy of both the accumulated point sum score and the accumulated betavalues demonstrated AUC of 0.82 . The Youden index indicate a cut off value $\geq 6$, at which DMV is predicted with a sensitivity of $0.85(0.81-089 ; 95 \% \mathrm{Cl})$ and a specificity of $0.61(0.61-0.62$; $95 \% \mathrm{Cl}$ ) (Table 2). These findings differ from the DIFFICAIR trial, where the individual clinicians' non-weighted prediction after assessment of the same risk-factors resulted in a sensitivity of 0.14 and a specificity of 0.99 .

Conclusion(s):

The weighted point score may prove a useful tool for predicting DMV. We will validate the model in all randomized patients using multiple imputation. 